\title{
Von Vielfalt und Einseitigkeit
}

\section{Von Anja Gregor}

Armin Nassehi (2012) fordert in seinem SozBlog-Eintrag vom 08. November 2012 über den 36. Kongress der Deutschen Gesellschaft für Soziologie (DGS) in Bochum: „Mehr Einseitigkeit, bitte!"Seine Begründung: Es gebe in der Soziologie keine Konflikte mehr. Es fehlten die Auseinandersetzungen innerhalb des Faches, die den Kongress erzählbar machten. Das Problem sei, dass Theorien bemüht seien, mit potenziell konfligierenden Theorien Harmonisierungen anzustreben, statt auf ihrer Einseitigkeit zu beharren und so Kontroversen zu provozieren, die das Fach in Bewegung hielten. Möglicherweise, so möchte ich hier versuchsweise argumentieren, handelt es sich dabei aber gar nicht um eine konfliktvermeidende Harmonisierung von streitbaren Theorien; die Schuld an der Konfliktarmut scheint viel mehr in der inneren Ausgeglichenheit der Soziologie zu suchen. Nassehis Kritik an den Friedensangeboten innerhalb der Disziplin verweist selbst auf ganz andere Aspekte, die konfliktvermeidend wirken können. Möglicherweise sind Mechanismen am Werk, die ,hinter dem Rücken der Agierenden' auch und gerade auf dem Kongress eine paradigmatische Harmonisierung der Soziologie vermitteln, indem einerseits die Ordnung des Diskurses reproduziert wird auf eine Weise, die ihn potentiell unbeweglich(er) macht. Wenn nämlich indirekt und unausgesprochen Regeln darüber wirken, wer was unter welchen Umständen sagen darf, sind Schließungsprozesse am Werk, die zur Erstarrung des Diskurses beitragen und die Draufsicht, also eine kritische Reflexion der Grenzen des Diskurses, erschweren oder gar verhindern. Beispielsweise, wenn beim zugegebenermaßen recht polemischen und ungewohnt lauten Eröffnungsvortrag von Daniel Cohn-Bendit mehrheitlich verdrehte Augen, kopfschüttelnde Ablehnung oder ignorierende Gleichgültigkeit beobachtet werden können, wird sich damit eines Innen versichert, das sich darüber einig ist, dass der, der da vorn spricht, das Außen ist, von dem sich abgegrenzt und gleichsam ex negativo als SoziologIn definiert werden kann. Wenn ein Diskurs sich derart abschließt, so ist nicht verwunderlich, dass von außerhalb kein Schwein guckt (Nassehi 2012). Andererseits, und das ließ sich auf dem Kongress in Bochum so verschiedentlich wie vielfältig beobachten, können potenziell unpässliche Strömungen innerhalb des Diskurses mit subtilen Mitteln marginalisiert werden. Jene Untersuchungsgegenstände, die aus politischer Korrektheit (und qua Motto) Teil des Kongresses sein müssen, aber nicht zum Oberflächenkonsens der Disziplin passen, können mit als Anerkennung getarnten Sprechakten repräsentiert und im weiteren Verlauf der Veranstaltung weitgehend ignoriert werden. Die Vielfalt drückte sich auf dem Kongress vor allem dadurch aus, dass der Zusammenhalt (oder die Harmonisierung) klassischer, tradierter und etablierter Themen in der Soziologie ,Randbezirke" produziert. Bereits Imke Schmincke schreibt über das Motto des DGS-Kongresses 2010 in Frankfurt am Main („Transnationale Vergesellschaftung“), Transnationalität hatte dort „mit Blick auf die Gesamtheit der Veranstaltungen [...] eher einen deskriptiven als einen systematischen Stellenwert" (Schmincke 2010: 111) - über den Kongress in Bochum ließe sich hinsichtlich des Mottos Vielfalt wohl Ähnliches behaupten. Den provokativen Blog-Eintrag Armin Nassehis zum Anlass nehmend, möchte ich in diesem Bericht im Zuge der Benennung erinnerungswürdiger Eckdaten und Ereignisse eine wissenschaftskritische Betrachtung vornehmen, die insbesondere eine Standortbestimmung von zweien jener Disziplinen innerhalb der Soziologie versucht, die ich hier als auf bestimmte subtile Weise marginalisiert oder nur mit Alibi-Nennungen repräsentiert beschrieben habe: die soziologische Geschlechterforschung / Gender Studies bzw. deren diskursive Erweiterung, die Queer Studies. Die folgenden Ausführungen arbeiten ganz bewusst methodisch mit Scheuklappen, um fokussiert eben jene Verhältnisse in den Blick zu nehmen, die durch die Gender und Queer Studies behandelt und / oder kritisiert werden. 


\section{Die Soziologie und die Geschlechterverhältnisse}

Ein Anliegen der sozialwissenschaftlichen Frauenforschung war seit den späten 1960er Jahren, den Androzentrismus der Inhalte ebenso wie der Organisation des Wissenschaftsbetriebes zu analysieren. Grundlegend war die Prämisse, dass Frauen weder Objekt noch Subjekt der Wissenschaft waren. Die Kritik war damit immer eine auf zwei Ebenen: Inhaltlich erweiterten feministische Theoretikerinnen die Darstellung und Diskussion soziologischer Klassiker um die Kategorie Geschlecht (beispielhaft genannt seien hier Simmel, Marx, Berger / Luckmann oder Foucault; ganz aktuell setzt sich der Sammelband ,Zeitgenössische Gesellschaftstheorien und Genderforschung" von Heike Kahlert und Christine Weinbach damit auseinander), organisatorisch forderten sie den gleichberechtigten Zugang von Frauen zur Wissenschaft (und realisierten damit beispielsweise die Institutionalisierung heutiger Gleichstellungsaufgaben an Universitäten und deren personale Repräsentation durch Gleichstellungsbeauftragte). Der Frauenanteil an den Professuren der Soziologie liegt laut Mau und Huschka heute bei circa 25\% der insgesamt 320 - jedoch besetzen Frauen vor allem W1- und W2-Professuren (Mau / Huschka 2010). Es gibt in der Soziologie 24 Professuren mit einer Voll- oder Teil-Denomination für Frauen- und Geschlechterforschung / Gender Studies in Deutschland (Stand: 23. Februar 2012), nur eine davon in den neuen Bundesländern (vgl. ZE für Förderung von Frauen- und Geschlechterforschung 2012).

Inwiefern also war die soziologische Geschlechterforschung - inhaltlich - auf dem Kongress vertreten, und in welcher Weise und wie häufig waren Frauen - organisatorisch - auf dem Kongress repräsentiert?

Im Eröffnungsvortrag des Kongresses ist lediglich in einer Aufzählung das Thema sexuelle Orientierung benannt - es wird herausgestellt, dass die eingetragene Lebenspartnerschaft ein Marker für die zunehmende Anerkennung von Vielfalt in Deutschland sei. Dass diese Nennung überhaupt geschieht, ist bemerkenswert; dass es aber im weiteren Verlauf des Kongresses wenig bis keine weitergehende Auseinandersetzung mit diesem Phänomen gibt, lässt sie zur Alibi-Benennung verkommen und verdeutlicht die marginalisierte Stellung der Inhalte der oben benannten Teildisziplinen innerhalb der Soziologie. Ausführungen über das Geschlechterverhältnis in Führungspositionen (oder etwas kontroverser: der Professuren in der Soziologie) hätten einen zunächst einmal organisatorischen Konflikt innerhalb der Soziologie zumindest einmal angedeutet, statt ihn zu verdrängen. In der Organisation des Kongresses sind Frauen zwar sichtbar, aber nicht gleichberechtigt. 8 der 12 Vorlesungen zum Mittag und Abend werden von Männern gehalten, das einzige Thema mit einem diversity-Ansatz bestreitet eine Frau. Und das ist denn auch die Stoßrichtung der Repräsentation von Geschlechterforschung auf dem Kongress: Wenn darüber vorgetragen, diskutiert und gesprochen wird, dann von den Betreffenden selbst. Geschlecht ist in der Soziologie, so zumindest vermittelt es das ansonsten vielseitige und spannende Programm, mitnichten eine Querschnittskategorie, deren Mitdenken sich auch jenseits der mit der Sektion Frauen- und Geschlechterforschung assoziierten Themen und Personen etabliert hätte. Jene Sektion scheint sich auf den ersten Blick - wie bedauerlicherweise viele Projekte zur Geschlechtergleichstellung- auf dem Kongress tendenziell nach außen zu schließen. Oder wird sie doch eher von außen geschlossen, abgeschirmt? Ist die Schließung möglicherweise eine Reaktion auf die fehlende Responsivität des restlichen soziologischen Diskurses? Oder auf den aus jahrzehntelanger Erfahrung heraus erwartbaren kalten Gegenwind, sobald es explizit und konkret um Geschlechter- und Gleichstellungsthematiken geht? Jene Personen, die sich zum Mitglieder-Treffen der Sektion versammeln, finden sich nahezu in gleicher Besetzung auch zur Buchpräsentation des oben genannten Sammelbandes ein, um (gem)einsam das gemeinsame Projekt zu feiern. Anerkennung von ,außen' gibt

es hier augenscheinlich wenig bis keine; die Abstimmung über die Bedeutung soziologischer Themen wird auf dem Kongress eben auch mit den Füßen vorgenommen. Die beobachtete 
Szene ist beispielhaft: Der potenzielle organisatorische Konflikt wird hier durch Marginalisierung (in Form von Nichtbeachtung) und Spezialisierung (auf eben jene, die sich sowieso damit beschäftigen) an den Rand der Aufmerksamkeit gedrängt. Der Mainstream kann sich auf die formale Präsenz der Geschlechterforschung berufen und ,seinen ' Betrieb der Disziplin großteilig unbehelligt von ungemütlichen Auseinandersetzungen mit der eigenen Geschlechterblindheit fortsetzen. Eine explizite Herausforderung jenes Konfliktes, beispielweise durch eine Plenumsveranstaltung zum Stand der Gleichstellungspolitik in der Soziologie in Deutschland, hätte möglicherweise einen jener - auch inhaltlichen - Konflikte provoziert, die sich Armin Nassehi in seinem Beitrag wünscht. So bleibt es aber bei der - natürlich wichtigen und richtigen - Podiumsdiskussion zum Ausstieg aus dem CHE-Ranking, die ein weiteres Mal die Geschlossenheit oder besser: den Zusammenhalt des Faches auch über Professionsgrenzen hinaus vorführt, wenn die recht zahlreich auf dem Kongress vertretenen Studierenden und Lehrende gemeinsam und fachkundig gegen die fadenscheinigen Argumente der CHE-MitarbeiterInnen halten.

Zurück zu den inhaltlichen Aspekten der diskutierten Themen auf dem Kongress. Neben den Veranstaltungen der Sektion Frauen- und Geschlechterforschung (oder ihrer einzelnen Mitglieder als OrganisatorInnen) finden sich das Plenum „Vervielfältigung der Familien- und Geschlechterarrangements“, die Ad-hoc-Gruppe „Soziologie der Sexualität“ und einzelne Vorträge, die Geschlechterverhältnisse unter bestimmten Aspekten und Geschlecht als intersektional verschränkte Querschnittskategorie in den Blick nehmen. Mit Blick auf das gesamte Programm ist die Geschlechterforschung jenseits der ,üblichen Verdächtigen“ marginal vertreten, queertheoretische Ansätze finden sich auch innerhalb jener Gruppen nur vereinzelt. So kündigt Ilse Lenz ihren Intersektionalitätsansatz als die sexuelle Orientierung berücksichtigend an, verbleibt im Vortrag dann aber doch in den vertrauten Gefilden des Spannungsfeldes von race, Klasse und Geschlecht. Die Hoffnung bleibt, dass sich die Berücksichtigung queertheoretischer Ansätze in der Sektion mit Uta Schirmer als neuer Vorstandsperson, wie von ihr angekündigt, tatsächlich ändert. In der Ad-hoc-Gruppe „Soziologie der Sexualität“ wiederum hat nur ein Vortrag Geschlechter- / Begehrensvielfalt und nicht heterosexuelles Sexualverhalten zum Inhalt. Und wenn jener Vortrag von Rüdiger Lautmann sich statt der angekündigten „Sexual diversity und Sozialtheorie“ die Ausbuchstabierung des Kürzels LSBTI zum Gegenstand nimmt und damit einen nicht mal mehr als Einführungsvortrag überzeugenden Beitrag liefert (der zudem an verschiedenen Stellen inhaltlich erschreckend daneben lag), dann ist dies ein mehr als trauriger Wasserstandsanzeiger zum Umgang mit und Wissen über die Forschungsgegenstände der Queer Studies in der Soziologie.

\section{Die Einseitigkeit der Vielfalt}

Der 36. DGS-Kongress in Bochum vermittelte weiterhin: Die Erkenntnisse und Entwicklungen der sozialwissenschaftlichen Queer und Gender Studies sind längst nicht angemessen im Mainstream (der weiterhin ein male stream ist) der Soziologie angekommen. Die Soziologie in Deutschland und damit die Sicht auf Vielfalt und Zusammenhalt ist weiterhin vor allem männlich und heterosexuell - und um kurz hinter den Scheuklappen hervorzuschielen und einen Blick auf die KongressteilnehmerInnen zu werfen: vor allem weiß, im besten Alter, finanziell gut situiert und / oder institutionell angebunden (wie sonst sollte eine/ $\mathrm{r}$ den horrenden Kongressbeitrag berappen können?). Und wenn sie weiblich und breit rezipiert ist, dann in den bereits bestehenden und etablierten (also tradiert-männlichen) Mustern. Frauen sind weiterhin vor allem dann erfolgreich, wenn sie die männliche Logik des Wissenschaftsbetriebes bedienen. Wer hingegen Frauen- und Geschlechterforschung/Queer Studies betreibt, verweist auf jene blinden, konflikthaften Stellen, denen sich etwa die soziologische Theorie weiterhin verschließt, wenn sie nicht anerkennt, dass es jenseits harmonisierender klassischer Theorien genug Ansatzpunkte für eine konflikthafte, interne Auseinandersetzung mit (queer)feministi- 
schen Theorien und Analysen gibt. Der Dialog über die Erkenntnisse der Geschlechterforschung, inhaltlich-theoretischer wie organisatorischer Natur, könnte die Grenzen der Soziologie möglicherweise wieder etwas fluider machen und damit die Spannung innerhalb des Feldes wieder erhöhen - vor allem aber würde er eine Reihe produktiver interner Konflikte provozieren, mit denen sich insbesondere die soziologische Theorie auseinanderzusetzen hätte.

\section{Literatur}

Kahlert, Heike / Christine Weinbach (2012): Zeitgenössische Gesellschaftstheorien und Genderforschung. Frankfurt / Main.

Mau, Steffen / Denis Huschka (2010): Die Sozialstruktur der Soziologie - Professorenschaft in Deutschland. Diskussionspapier des Wissenschaftszentrums Berlin für Sozialforschung, abrufbar unter http:// skylla.wz-berlin.de/pdf/2010/i10-204.pdf, letztes Abrufdatum 6.12.2012.

Nassehi, Armin (2012): Mehr Einseitigkeit, bitte! Beitrag zum SozBlog der DGS am 8. November 2012. Abzurufen unter: http://soziologie.de/blog/?p=805, letztes Abrufdatum: 6.12.2012.

Schmincke, Imke (2011): Transnational und doch lokal. Bericht vom 35. Kongress der Deutschen Gesellschaft für Soziologie, 11.-15. Oktober 2010 in Frankfurt am Main, in: Soziale Welt $62 / 1$, S. 109-114.

Zentraleinrichtung zur Förderung von Frauen- und Geschlechterforschung (2012): Professuren mit einer Teil- oder Voll-Denomination für Frauen- und Geschlechterforschung / Gender Studies an deutschsprachigen Hochschulen, abrufbar unter http://www.zefg.fu-berlin.de/datensammlung/genderprofessuren/index.html, letztes Abrufdatum: 6.12.2012.

Anja Gregor, M.A. Friedrich-Schiller-Universität Jena Institut für Soziologie Carl-Zeiß-Straße 2 07743 Jena anja.gregor@uni-jena.de 